\title{
Electromiografía de Superficie y Articulografía Electromangética para Análisis de Dimensión Vertical. Reporte de Protocolo
}

\section{Surface Electromyography and Electromagnetic Articulography to Analyze VerticalDimension. Protocol Report}

\author{
J. Gómez*, F. Lezcano**, V. Peldoza***, R. Fuentes**
}

\section{RESUMEN}

Introducción: La correcta determinación de la Dimensión Vertical (DV) es esencial al momento de rehabilitar a un paciente. En la literatura existen escasas investigaciones que relacionen en conjunto la DV con otras variables como actividad electromiográfica.El objetivo de este estudio es describir una técnica para el análisis de la DVa través del Espacio Libre de Inoclusión (ELI), mediante la utilización de articulografíaelectromagnética y electromiografía de superficie.

Material y Métodos: Se registró la DV mediante el uso de un articulógrafo electromagnético AG501 simultáneamente con la actividad electromiográfica de músculos masetero y temporal mediante la utilización de electromiógrafo de superficie EMG VIII, según protocolo de DVO y DVP.El estudio se realizó en el Laboratorio de Fisiología Oral del Centro de Investigación en Ciencias Odontológicas (CICO) con la Aprobación del Comité Ético Científico de la Universidad de La Frontera.

Resultados: Se obtuvo un protocolo para el uso de electromiografía y articulografía electromagnética 3D en el análisis de dimensión vertical, el cual permite caracterizar la dimensión vertical mediante una medición de distanciavertical, medición de distancia 3D, ángulo de apertura y la actividad eléctrica de los músculos elevadores de la mandíbula a distintos grados de apertura.

Conclusión: El protocolo obtenido puede ser utilizado con el fin de generar recomendaciones clínicas para rehabilitar a pacientes.

PALABRAS CLAVE: Electromiografía de Superficie, Articulografía Electromangética, Dimensión Vertical, Oclusión.

\section{ABSTRACT}

Introduction: The correct determination of the Vertical Dimension (VD) is essential when rehabilitating a patient. There is little research in the literature that collectively relates the VD to other variables such as electromyographic activity. The objective of this study is to describe a technique for the analysis of the VD through the Interocclusal Distance, using electromagnetic articulography and surface electromyography.

Material and Methods: The VD was recorded by using an AG501 electromagnetic articulgraph simultaneously with the electromyographic activity of masseter and temporal muscles using EMG VIII surface electromyograph, according to DVO and DVP protocol. The study was carried out in the Oral Physiology Laboratory of the Center for Research in Dental Sciences (CICO) with the approval of the Scientific Ethical Committee of the University of La Frontera.

* Programa de Magíster en Odontología, Facultad de Odontología, Universidad de La Frontera, Temuco, Chile.

* * Centro de Investigación en Ciencias Odontológicas (CICO), Departamento de Odontología Integral Adultos,

Facultad de Odontología, Universidad de La Frontera, Temuco, Chile.

*** Estudiante, Facultad de Odontología, Universidad de La Frontera, Temuco, Chile. 
Results: A protocol was obtained for the use of electromyography and 3D electromagnetic articulography in the vertical dimension analysis, which allows the vertical dimension to be characterized by a vertical distance measurement, 3D distance measurement, opening angle and the electrical activity of the lifting muscles of the jaw to different degrees of opening.

Conclusion: The proposed protocol could be used to generate clinical recommendations to rehabilitate patients.

KEY WORDS: Surface Electromyography, Electromagnetic Articulography, Vertical Dimension, Occlusion.

Fecha de recepción: 1 de octubre de 2019

Fecha de aceptación: 2 de octubre de 2019

J. Gómez, F. Lezcano, V. Peldoza, R. Fuentes. Electromiografía de Superficie y Articulografia Electromangética para Análisis de Dimensión Vertical. Reporte de Protocolo. 2020; 36, (3): 151-159.

\section{INTRODUCCIÓN}

El objetivo de la rehabilitación oral es restablecer la armonía y función de la cavidad oral cuando ha habido pérdida de dientes, tejidos u otras estructuras orales, por medio de la confección de elementos que reemplacen las estructuras perdidas.

La dimensión vertical es un concepto clínico utilizado en el área de rehabilitación oral, que indica la altura del tercio inferior de la cara ${ }^{(1,2)}$. La correcta determinación de la dimensión vertical es esencial al momento de rehabilitar a un paciente para devolverle la normofunción y estética.

La dimensión vertical varía de acuerdo a las diferentes posiciones que puede adoptar la mandíbula en el plano vertical (determinada por los distintos procesos como masticación, deglución, fonación respiración entre otros) ${ }^{(3)}$.

Desde el punto de vista clínico y para objeto de rehabilitación encontraremos dos dimensiones importantes; Dimensión Vertical Oclusal y la Dimensión Vertical Postural.

La dimensión vertical oclusal (DVO) es la altura del tercio inferior de la cara cuando la persona se encuentra en posición de máxima intercuspidación" ${ }^{(1,4)}$.

La dimensión vertical postural (DVP) es la altura del tercio inferior de la cara cuando la persona se encuentra en posición postural mandibular (PPM) ${ }^{(1)}$. PPM se define como la posición que la mandíbula asume en relación a la maxila cuando la persona se encuentra en posición cómoda y ortostática, con un contacto labial suave y dientes en inoclu- sión. Es una posición básica de la cual parten los movimientos funcionales de la mandíbula ${ }^{(1-3)}$.

La dimensión vertical se puede evaluar a través del espacio libre de inoclusión o llamado también espacio de inoclusión fisiológico (ELI). ELI se define como el espacio que se forma entre las superficies oclusalesentre la mandíbula y la maxila cuando la mandíbula se encuentra en posición postural mandibular o de descanso ${ }^{(5,6)}$, encontrándose un espacio interoclusal entre 2 a $4 \mathrm{~mm}^{(5-7)}$.

El ELI se obtiene mediante el cálculo de la diferencia en milímetros entre la DVP y la DVO. La incorrecta determinación de este produce problemas estéticos (labios con dificultad de contacto al cierre hipertónicos, labios hipotónicos), funcionales (dificultad de habla y masticación), contracciones musculares excesivas, queilitis angular, daño en dientes, periodonto y tejidos de soporte, trastornos temporomandibulares, por lo que es e gran importancia reestablecer un ELI adecuado en pacientes que han perdido sus dientes con el propósito de reestablecer DVO ${ }^{(3,8)}$.

Dentro de los métodos para determinar el espacio libre de inoclusión fisiológico encontramos ${ }^{(5)}$ :

Apariencia general, opinión del paciente, métodos métricos (Ângulo Gonial mandíbula, Willis Gauge), obtención de PPM (método de deglución, test de habla).

Como se ha descrito anteriormente se podrá obtener ELI a través de la obtención PPM.

En la literatura existe una amplia variedad de estudios que hablan de la obtención de PPM, los 
cuales varían según la metodología utilizada.

Entre los más populares encontramos los métodos fonéticos donde diversos autores con el fin de reestablecer DVO en pacientes desdentados exponen que la pronunciación de diversos fonemas sirve para la obtención de posición de reposo, PPM o DVP a través del Closest speaking space(CSS) y esto a su vez para el restablecimiento de DVO (9-14). El método sin comando, es uno de los métodos más utilizados por su facilidad de ejecución y simpleza, este método consiste en pedirle al individuo que mire un punto fijo, estando cómodo, relajado, con los dientes en inoclusión y un leve contacto labial. El método de deglución, distintos investigadores concluyen que es un método efectivo para determinar la DVP(15-16), el cual se basa en que el ciclo de deglución comienza cuando la mandíbula se desplaza desde la posición de reposo hasta que se produce un ligero contacto entre los dientes, después de lo cual la mandíbula regresa a la posición de reposo.

\section{Importancia de la actividad electromiográfica en} la evaluación de Dimensión Vertical

La electromiografía es "el registro de las variaciones de voltaje que se producen en las fibras musculares como expresión de la despolarización de sus membranas durante la contracción espontánea o voluntaria" ${ }^{(17)}$.

Se han realizado múltiples estudios donde se intenta determinar la posición postural mandibular con actividad electromiográfica.

Garnick y Ramfjord ${ }^{(18)}$ en 1962 realizaron un estudio con EMG midiendo la actividad de los músculos temporales, digástricos y maseteros obteniendo que la posición de reposo (obtenida por métodos fonéticos y de deglución) no se condice con la actividad mínima de los músculos, mas bien ésta era a un grado de apertura mayor, siendo el rango promedio para éstos músculos de $11 \mathrm{~mm}$.

En 1981 Rugh y Drago ${ }^{(19)}$ utilizaron un kinesiógrafo para determinar la actividad eléctrica mínima muscular relacionada con las distintas alturas faciales en distintos músculos mandibulares en hombres y mujeres, obteniendo que la medida promedio para posición de reposo clínica o PPM de $2.1 \mathrm{~mm}$ y mínima actividad electromiográfica promedio para hombres y mujeres de $8.6 \mathrm{~mm}$, siendo la diferencia entre estas dos posiciones de $6 \mathrm{~mm}$ en promedio.
Michelotti en $1997^{(20)}$ compara la posición postural de reposo clínico y electromiográfica en pacientes con características morfológicas faciales distintas obteniendo que al variar la dimensión vertical aumentándola, la actividad electromiográfica disminuía. Por lo que no se condice la posición clínica de descanso con la electromiografía inactiva o posición de descanso muscular. Además sugiere en su estudio que "la musculatura de la mandíbula era mínimamente activa en un rango de posiciones verticales en lugar de en un punto específico".

Manns et al, en $1981^{(21)}$ encontraron una mínima actividad eléctrica muscular en un rango de 9 a $10 \mathrm{~mm}$ para el músculo masetero, mientras que, la mínima actividad EMG para músculo temporal anterior fue de 6 a $19 \mathrm{~mm}$.

Por otra parte, Michelotti et al, encontraron que el valor promedio para la mínima actividad EMG era de $7.7+-2.7 \mathrm{~mm}$ y la DVP o posición de reposo era de $1.4+-1.1 \mathrm{~mm}$. La diferencia promedio entre la EMG y la posición de reposo clínico fue de $6.3 \pm 2.5 \mathrm{~mm} .{ }^{(20)}$.

Hoy en día se concluye que la posición de resposo o postural mandibular es un concepto distinto a la posición de menos actividad electromiográfica y que sí existe actividad electromiográfica de los músculos elevadores de la mandíbula en posición postural mandibular donde existe un espacio libre de inoclusión entre 1 a $3 \mathrm{~mm}$ ya que los músculos elevadores de la mandíbula ayudan a contrarrestar la fuerza de gravedad que tiende a deprimir la mandíbula ${ }^{(1,18-22)}$. Además, se encontró que la mínima actividad eléctrica muscular de los músculos elevadores de la mandíbula se encuentra en un rango entre los 8 a $12 \mathrm{~mm}$ de espacio de inoclusión, y que luego la actividad electromiográfica aumenta gradualmente hasta apertura máxima ${ }^{(1,22)}$.

Los estudios existentes utilizan los métodos de obtención de dimensión vertical anteriormente descritos, sin embargo, la mayoría de éstos emplean instrumentos poco precisos y metodologías poco estandarizables lo cual podría producir distorsión en los registros. Estas dificultades metodológicas han sido superadas con la Articulografía Electromagnética (EMA), que representa un método seguro, no invasivo y preciso al momento de evaluar los movimientos mandibulares (23-25). 


\begin{tabular}{lccccccccccc} 
& \multicolumn{1}{c}{ TABLA 1. Datos de registro de un sujeto de prueba } \\
& Apertura & \multicolumn{4}{c}{ Distancia 3D $(\mathrm{mm})$} & \multicolumn{1}{c}{ Distancia vertical $(\mathrm{mm})$} & \multicolumn{1}{c}{ SEMG } & \multicolumn{2}{c}{ RMS $(\mu \mathrm{V})$} \\
& $\left({ }^{\circ}\right)$ & Der & Cen & Izq & Der & Cen & Izq & MD & MI & TD & TI \\
PPM Método sin comando & 1,4 & 2,2 & 2,7 & 2,3 & 2,1 & 2,7 & 2,2 & 1,9 & 2,8 & 6,0 & 3,3 \\
PPM Método deglución & 0,7 & 1,3 & 1,9 & 1,8 & 1,0 & 1,3 & 1,3 & 1,9 & 2,6 & 2,6 & 2,7 \\
\hline
\end{tabular}

TABLA 2. Datos de registro de un sujeto de prueba a distintas aperturas

\begin{tabular}{|c|c|c|c|c|c|c|c|c|c|c|}
\hline \multirow{2}{*}{$\begin{array}{c}\text { Apertura } \\
\left({ }^{\circ}\right)\end{array}$} & \multicolumn{3}{|c|}{ Distancia 3D (mm) } & \multicolumn{3}{|c|}{ Distancia vertical $(\mathrm{mm})$} & \multicolumn{2}{|c|}{ SEMG } & \multicolumn{2}{|c|}{ RMS $(\mu \mathrm{V})$} \\
\hline & Der & Cen & Izq & Der & Cen & Izq & MD & MI & TD & TI \\
\hline 2,2 & 3,1 & 3,8 & 3,3 & 2,6 & 3,5 & 2,9 & 1,9 & 2,8 & 2,8 & 3,0 \\
\hline 2,5 & 5,3 & 5,7 & 4,4 & 5,3 & 5,7 & 4,4 & 1,8 & 2,6 & 12,2 & 3,3 \\
\hline 5,4 & 9,1 & 10,6 & 8,3 & 9,1 & 10,4 & 8,0 & 1,7 & 2,7 & 2,9 & 2,9 \\
\hline 6,8 & 9,4 & 12,2 & 10,4 & 9,2 & 12,0 & 10,2 & 1,9 & 2,7 & 6,6 & 3,7 \\
\hline 8,0 & 12,9 & 15,8 & 12,9 & 12,8 & 15,7 & 12,8 & 1,9 & 2,8 & 6,2 & 3,4 \\
\hline 10,8 & 14,1 & 18,5 & 15,4 & 14,1 & 18,4 & 15,3 & 2,0 & 2,9 & 5,2 & 3,5 \\
\hline 12,6 & 16,1 & 21,1 & 17,2 & 15,9 & 20,9 & 17,0 & 2,1 & 2,9 & 5,3 & 3,7 \\
\hline 14,4 & 18,1 & 23,6 & 19,1 & 17,8 & 23,3 & 18,7 & 2,2 & 3,0 & 5,4 & 4,0 \\
\hline 17,4 & 19,8 & 26,7 & 21,4 & 19,5 & 26,4 & 21,1 & 2,2 & 3,2 & 2,5 & 3,2 \\
\hline 22,7 & 23,9 & 32,4 & 25,1 & 22,9 & 31,2 & 23,8 & 2,7 & 4,0 & 3,0 & 3,2 \\
\hline 25,2 & 25,2 & 34,9 & 27,1 & 23,6 & 33,2 & 25,6 & 11,6 & 19,4 & 19,1 & 4,3 \\
\hline 27,1 & 28,2 & 38,2 & 28,9 & 26,8 & 36,4 & 27,4 & 14,1 & 19,5 & 26,1 & 8,5 \\
\hline
\end{tabular}

Por lo anteriormente expuesto, el objetivo de este artículo es describir unatécnica para el análisis de la Dimensión Vertical a través del ELI mediante la utilización de Articulografíaelectromagnética 3D y electromiografía de superficie en músculos Temporal (ramas anteriores) y Masetero de forma simultánea.

\section{MATERIAL Y MÉTODOS}

Se realiza un reporte de técnica con un sujeto, el cual firmó un consentimiento informado.

Se registró simultáneamente la posición de la mandíbula mediante un articulógrafo electromagnético 3D (AG501, Carstens Medizinelektronik, Bovenden, Alemania) con una frecuencia de muestreo de 250 $\mathrm{Hz}$ y la actividad electromiográfica mediante un electromiógrafo de superficie (sEMG VIII, ArtOficio, Santiago, Chile) con una ganancia de 1000 .

\section{Descripción del articulógrafo electromagnético AG501} El articulógrafo electromagnético 3D AG501 desarrollados por CarstensMedizinelektronikes un dispositivo con nueve bobinas transmisoras que mediante la generación de campos electro magnéticos alternativos a distintas frecuencias permite la grabación digital, recolección y procesamiento de datos en tres dimensiones (3D). (24-25) El articulógrafo electromagnético ha sido certificado por la Comisión Federal de
Comunicaciones de Estados Unidos como undispositivo de comunicación de baja potencia, el cual utiliza campos electromagnéticos con un rango de frecuencia entre 7,5 y $13,75 \mathrm{KHz} .^{(24)}$

\section{Descripción del electromiógrafo de superficies EMG VIII.}

La electromiografía de superficie se basa en la conducción de potenciales eléctricos en la superficie cutánea o subcutánea proveniente de los músculos. El software de adquisición y análisis de datos electromiográficos del EMG VIII considera 8 canales de adquisición, poseeuna frecuencia de muestreo única de $1311 \mathrm{~Hz}$ y ganancias disponibles de 500, 1000 y 5000.

Para el estudio se utilizará una ganancia de 1000 .

\section{Previo al registro, preparación}

Este estudio cuenta con la aprobación del Comité ÉticoCientífico de La Universidad de La Frontera, Temuco, Chile (Folio N059_18). Fue realizado de acuerdo a los estándares internacionales de estudios en seres humanos.

Se realizó una preparación de los equipos EMA y EMG previo a los registros, está se realizó mediante la siguiente secuencia:

1.- Se encendió el EMA 15 minutos previos al registro 
y se conectaron los sensores númerados y calibrados en sus respectivas posiciones.

2.- Se ubicaron 3 sensores en un biteplane en punto derecho, centro e izquierdo y se fijaron con cinta adhesiva. (Fig. 1)

3.- Se encendió EMG y se conectaron los 4 sensores numerados a utilizar en su canal correspondiente y el sensor de referencia.

4.- Se configuraron los parámetros de EMG en el computador, quedo el equipo listo para las pruebas de registro.

5.- Se indicó al participante que en caso de portar elementos electrónicos y/o metálicosconductores de electricidad(celular, mp4 Aros, pulseras, reloj, etc.) los remueva con el fin e evitar interferencias en los registros.

6.- Se le solicitó al participante se siente bajo el cabezal del EMA en posición cómoda, erguido y mirando hacia el frente.

7.- Se limpiaron todas las zonas donde van a ir ubicados los sensores con gasa y alcohol.

8.- Se ubicaron los sensores EMA de referencia en el participante. Para ello se colocó adhesivo sobre el sensor e inmediatamente después se ubicaron (en puntocutáneo de mastoides derecho e izquierdo y glabela). (Fig. 2)

Con el fin de que estos sensores semantengan inmóviles durante los movimientos realizados por el participante se fijaron sus cables con cinta adhesiva a la piel. Esto se realiza con el fin de eliminar el movimiento de la cabeza del participante y obtener el movimiento aislado de la mandíbula con respecto al cráneo. Estos fueron identificados por EMA como los sensores que indican la localización espacial en los tres planos de la cabeza ${ }^{(24)}$.

Todos los sensores fueron adheridos a las superficies cutáneas y mucosas mediante un adhesivo tisular (Epiglu®, Alemania)

9.- Se colocó el conector de referencia en la muñeca del participante. Previo a su colocación, el contacto metálico se cubrió con una película de gel conductor y piel de la región de contacto se higienizó frotando gasa con alcohol.

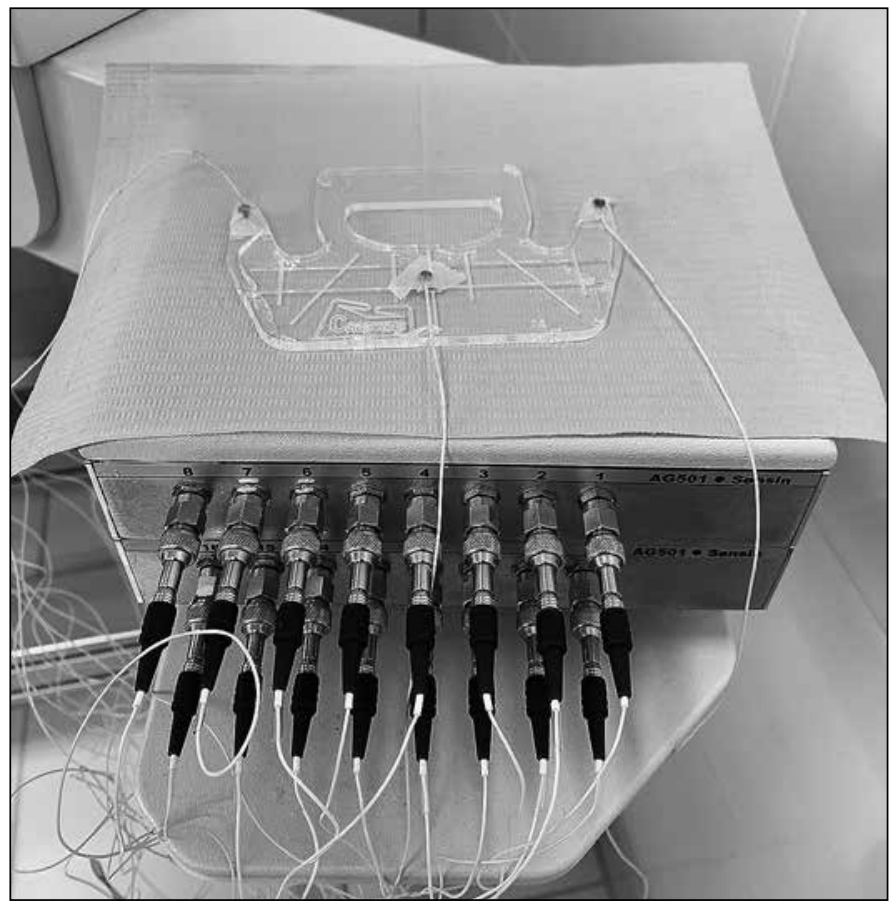

Figura 1. Biteplane con sensores posicionados en punto derecho, centro e izquierdo.

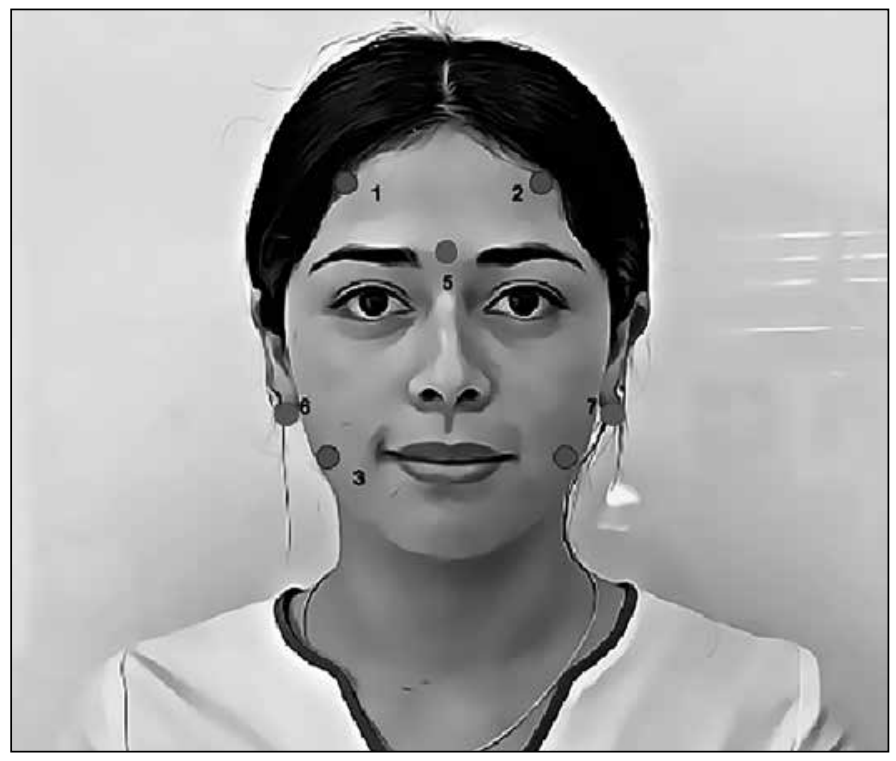

Figura 2. Distribución de los sensores de EMG y EMA extraorales colocados en el participante en puntos específicos. Sensores EMG: punto en piel músculo temporal anterior derecho (1) e izquierdo (2), punto en piel músculo masetero derecho (3) e izquierdo (4). Sensores EMA: glabela (5), punto en la piel de mastoides derecho (6) e izquierdo (7) (detrás de las orejas).

10.- Se ubicó el biteplane al que previamente se le colocaron los sensores en la boca del participante, y se le solicitó a éste que lo mordiera para posteriormente acomodarlo y que quede paralelo al plano Oclusal (con el objetivo de obtener el 
origen de coordenadas coincidentes con el plano oclusal del sujeto).

11.- Se Ajustó el cabezal del EMA y simultáneamente se visualizó en tiempo real el movimiento de los sensores (Se debe asegurar que todos los sensores estén dentro del área de medición en EMA).

\section{Registros de referencia y colocación de sensores activos EMA.}

Para el registro de referencia de EMA primeramente se realizó un registro de 5 segundos con el biteplane en la boca. Luego se retiró el biteplane de la boca del participante y se procedió a realizar "Init Head Correction", en el cual se seleccionan en el computador los sensores del biteplane y de referencia colocados en el participante, con el fin de que quede establecido el plano oclusal del participante y asignados los puntos de referencia inmóviles.

Posterior a esto, con la finalidad de tener un campo visual más amplio y facilita el acceso a la zona premolar y molar se le colocó un separador de labios al participante y se ubicaron los sensores de EMA activos (a $12 \mathrm{~mm}$ mínimo de distancia para entre ellos para que no que no haya interferencia) en el participante en línea media inter-incisiva inferior de la mandíbula, línea media entre segundo premolar y primer molar inferior derechos e izquierdos (Fig. 3). Para ubicar los sensores se secó previamente la zona donde fue ubicado cada sensor con una gasa.

Se seleccionaron los sensores activos EMA en el computador.

\section{Registros de referencia y} colocación de sensores activos EMG.

Para el registro de referencia de EMG se acondicionaron los sensores poniendo cinta doble faz en ellos y gel conductor en los contactos metálicos. Se procedió palpar la zona donde fueron ubicados los sensores para identificar la ubicación de las fibras musculares y se limpió con gasa y alcohol la zona.

Se colocaron los sensores de EMG paralelos a la fibra muscular de músculos maseteros y temporales anteriores (contactos metálicos deben ir en sentido perpendicular a la fibra muscular) (Fig. 2) y se colocó el sensor de referencia de EMG en codo en zona libre de fibras musculares.

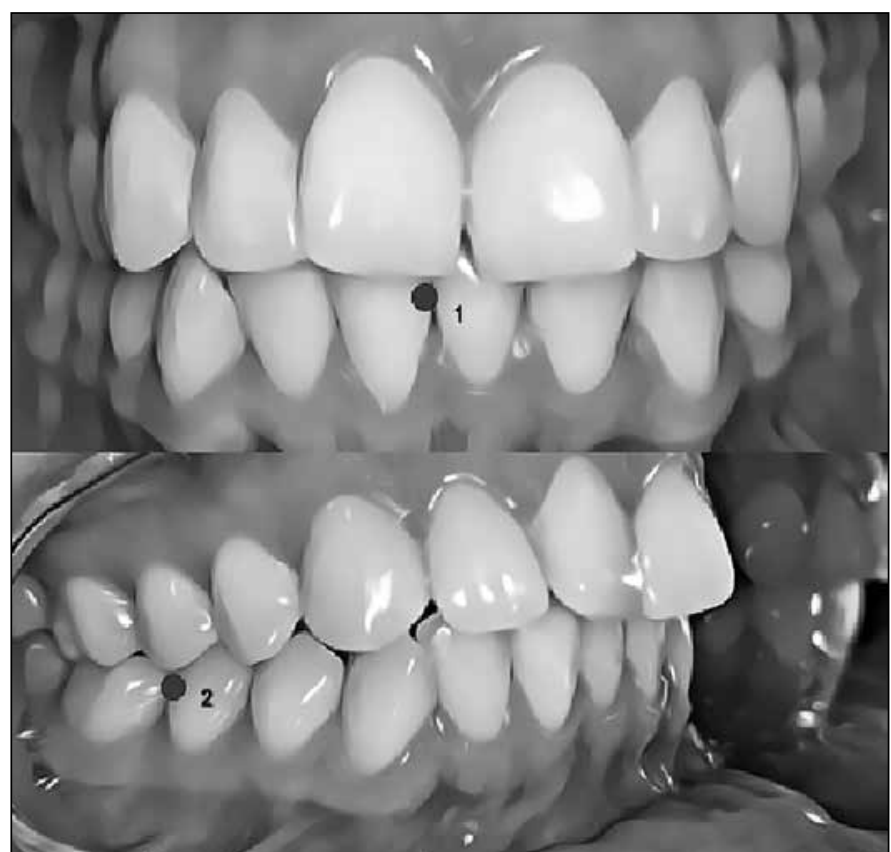

Figura 3. Distribución de los sensores de EMA intraorales colocados en puntos específicos: línea interincisiva inferior (1), línea interdental entre segundo premolar y primer molar inferior izquierda (2).

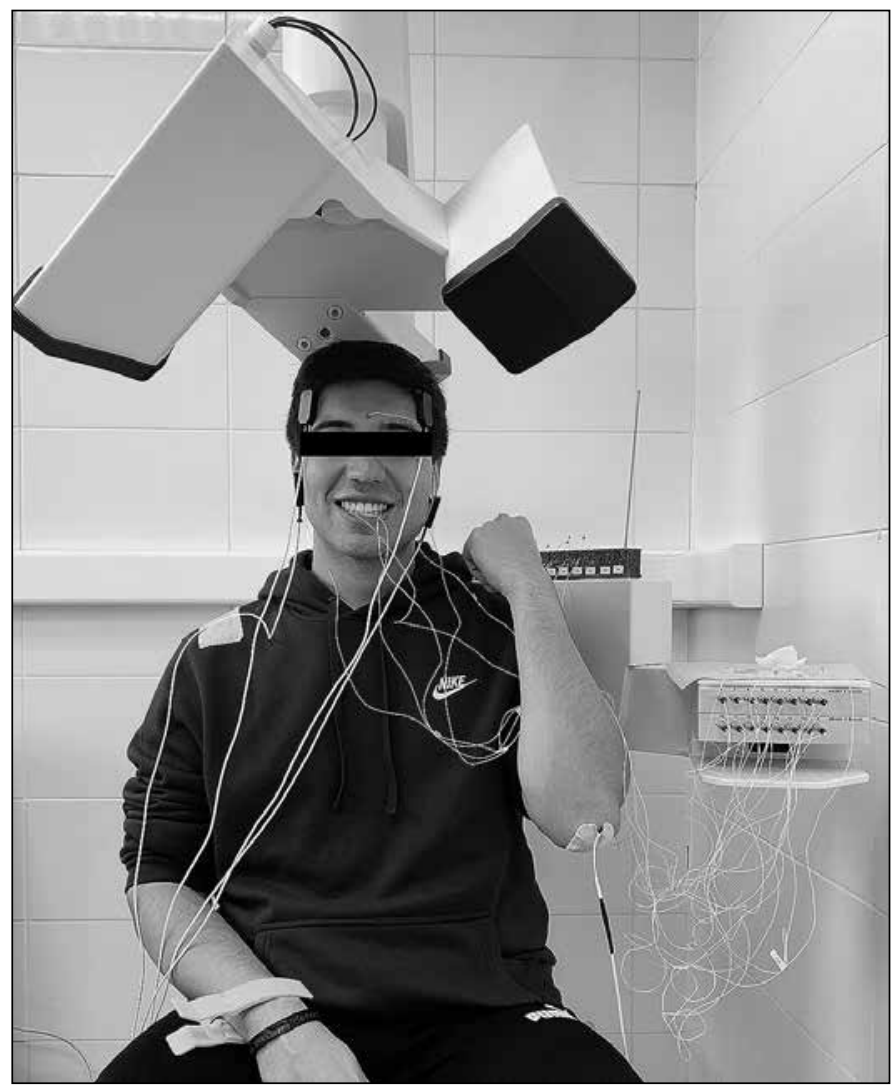

Figura 4. Sujeto de registro ubicado bajo cabezal EMA para medición, con sensores extraorales de EMA y EMG activos y de referencia ubicados respectivamente y sensores de EMA intraorales. 
Se realizaron las siguientes pruebas:

-Medición en reposo (Se reprodujo PPM).

- Máximo apriete voluntario (Se solicitó al participante que mordiera lo más fuerte que le sea posible).

- Apertura contra resistencia (Se solicitó al participante que intentara abrir la boca mientras otra persona aplica resistencia contra apertura).

Una vez terminadas las pruebas de registro y la colocación de los sensores en el participante, se procedió a realizar las mediciones simultáneas.

\section{Registro simultáneo.}

Una vez ubicado el participante bajo el EMA con todos los sensores de EMA y EMG, tanto activos como de referencia y habiendo realizado ambos registro de referencia se procedió a realizar los registros simultáneos de EMA Y EMG (Fig.4).

\section{Protocolo DVO}

Para el registro de la DVO se le indicóal participante que reproduzca la posición de máxima intercurspidación. Para lo ello, se le indicó al sujeto que mantenga la vista al frente en un punto fijo con un contacto dentario voluntario, leve y sin realizar fuerza de apriete. Esto se realizó con el fin de registrar el punto inicial del cual partirán el resto de los movimientos mandibulares.

\section{Protocolo DVP}

Para el registro de la DVP se reprodujo la posición postural mandibular mediante dos métodos.

a) Método sin comando: Se indicó al participante que mantenga la mirada al frente en un punto fijo, cómodo, relajado, dientes en inoclusión y labios en leve contacto.

b) Método de deglución: Se le pidió al participante que mire al frente y trague saliva. La posición final que adoptó la mandíbula después de la deglución fue la posición postural mandibular.

\section{Protocolo mediciones a distintas aperturas orales}

El registro se realiza partiendo de DVO (PMI) hasta apertura máxima del participante

Se le indicó al participante que mire al frente, vista fija y en posición cómoda.

Se realizaron mediciones cada 3 milímetros partiendo de DVO (PMI) hasta apertura máxima. Para obtener una distancia de $3 \mathrm{~mm}$ se visualizó en tiempo

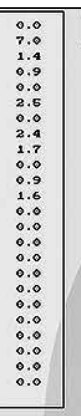

$f$

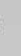

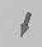

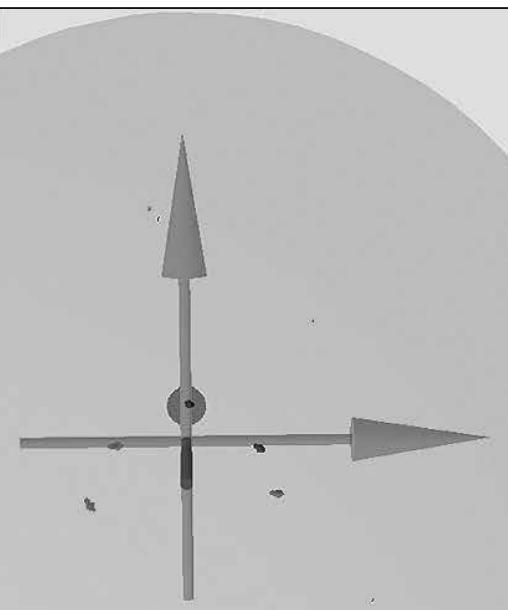

Nota: Cada registro se realizó 2 veces de manera continua. El sujeto en cada registro mantuvo la posición 5 segundos.

\section{Análisis de datos}

Los sensores de EMA producen un campo magnético alterno con diferentes frecuencias, esto induce un voltaje AC sobre cada sensor y detecta el recorrido efectuado de cada sensor obteniendo las coordenadas espaciales, determinando así su posición ${ }^{(25-26)}$.

Los datos resultantes se exportaron a un computador 


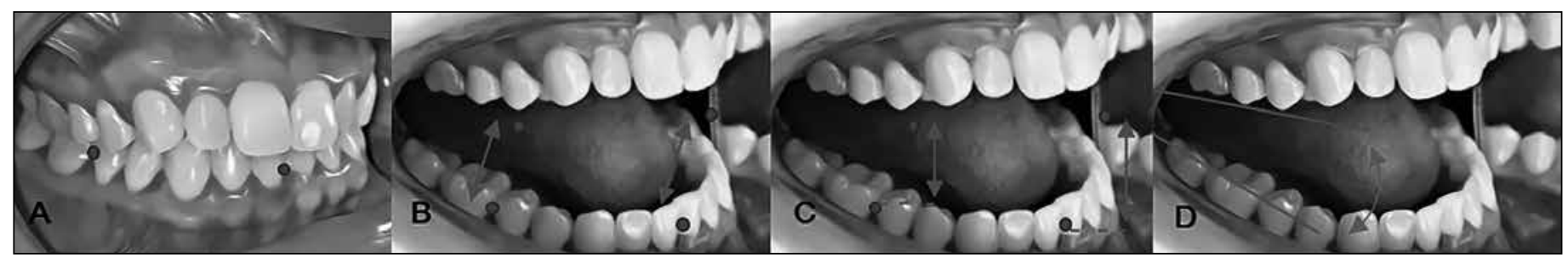

Figura 6. Parámetros evaluados con EMA desde posición de máxima intercuspidación (PMI) (A), (B) Distancia 3D, (C) Distancia vertical eje Z, (D) Ángulo de apertura.

donde fueron almacenados a través de archivos binarios (.pos). Estos se procesaron a través de rutinas de cálculo específicas o scripts, las cuales se basan en cálculo vectorial. Estos Scrips fueron desarrollados previamente para este estudio.

Los archivos resultantes de cada registro fueron procesados con Matlab (TheMathWorks, Inc., Natick, MA, EE. UU) a través estos Scripts.

Matlab es un "software computacional utilizado para aprendizaje automático, procesamiento de señal, procesamiento de imágenes, visión por ordenador, comunicaciones, diseño y otras funciones".

A su vez, los sensores EMG captan los potenciales eléctricos en la superficie de la piel y se expresan en ventanas de adquisición de datos en el computador EMG. Una vez terminada la adquisición se selecciona un trozo de la señal para calcular y mostrar el espectro en frecuencia (mediante FFT) y el RMS (Root Mean Square) del trozo seleccionado.

Los datos seleccionados de todos los canales activos fueron exportados y almacenados juntos en un único archivo. Estos datos posteriormente son procesados con Matlab para obtener diferentes parámetros ${ }^{\text {(Fig. 6) }}$.

\section{RESULTADOS}

La técnica permitió evaluar de manera eficaz y sencilla los parámetros distancia 3D $(\mathrm{mm})$, distancia vertical (desplazamiento sensor en eje $\mathrm{Z}$ ) $(\mathrm{mm})$, ángulo de apertura $\left({ }^{\circ}\right)$, EMG RMS $(\mu \mathrm{V})$ en cada posición adoptada por el sujeto (Masetero Derecho, MD; Masetero Izquierdo, MI; Temporal Derecho, TD; Temporal Izquierdo, TI) de forma simultánea con EMA y EMG (Fig. 6).

\section{CONCLUSIONES}

Con esta técnica se logró la obtención de registros de
EMA y EMG de manera simultánea para el estudio de dimensión vertical. Además, permitió medir los mismos parámetros (EMG RMS, distancia 3D, distancia lineal y ángulo de apertura) en distintas aperturas orales.

Con la utilización del Método sin comando y Método de deglución se logra obtener PPM y ELI.

Se obtuvo una técnica sencilla y reproducible, la cual permitirá la evaluación y estudio de la dimensión vertical y los distintos parámetros mencionados en otros estudios de fisiología oral.

\section{AGRADECIMIENTOS}

El presente trabajo fue financiado por la Dirección de Investigación, Universidad de La Frontera y forma parte del proyecto DI19-0087

\section{BIBLIOGRAFÍA}

1. Manns A, Díaz G. Sistema estomatognático. Universidad de Chile: Santiago de Chile; 1988.

2. Mack MR. Vertical Dimension: A dynamic concept based on facial form and oropharyngeal function. J ProsthetDent 1991; 66(Pt 4):478-85.

3. Jorquera C.Determinación de la dimensión vertical oclusal a través de la distancia clínica ángulo externo del ojo al surco tragus facial y la distancia radiográfica reborde externo de la órbita al conducto auditivo externo [Tesis].Santiago de Chile: Universidad de Chile; 2008.

4. Ferro KJ, Morgano SM, Driscoll CF, Freilich MA, Guckes AD, Knoernschild KL, McGarry TJ. The glossary of prothodontics terms. J Prosthet Dent. 2017; 117(Pt 5):90.

5. Johnson A, Wildgoose DG, Wood DJ. The determination of freeway space using two different me- 
thods. J Oral Rehabil 2002; 29(Pt 10):1010-3

6. Pleasure MA. Correct vertical dimension and freeway space. J Am DentAssoc 1951;43(Pt 2):160-3.

7. Farias-Neto A, Dias AHM, de Sousa SA, de Araújo CPD, de S Silva R. An investigation of the freeway space and facial proportions in dentate subjects. $\mathrm{J}$ Dent App 2014; 1(Pt 6):116-8.

8. Ramirez LM, Echeverría P, Zea FJ,Ballesteros LE. Dimensión vertical en edentados: relación con síntomas referidos. Int J Morphol 2013; 31(Pt 2):67280.

9. Alhajj MN, Khalifa N, Abduo J, Amran AG, Ismail IA. Determination of occlusal vertical dimension for complete dentures patients: an updated review. J Oral Rehabil2017; 44(Pt 11):896-907

10. Silverman MM. Accurate measurement of vertical dimension by phonetics and the speaking centric space. Part I. Dent Dig 1951; 57(Pt 6):261-5.

11. Silverman MM. Accurate measurement of vertical dimension by phonetics and the speaking centric space. Part II. Dent Dig 1951; 57(Pt 7):308-11.

12. Silverman $M M$. The speaking method in measuring vertical dimension. J ProsthetDent 1953; 3(Pt 2):193-9.

13. Burnett CA, Clifford TJ. Closest speaking space during the production of sibilant sounds and its value in establishing the vertical dimension of occlusion. J Dent Res 1993; 72(Pt 6):964-7.

14. Igic'c M, Kruni Nc'c, Aleksov L, Kostic'c M, Igic'c A, Petrovic'c MB, Dacic'c S, Igic'c S, Igic'c A. Determination of vertical dimension of occlusion by using the phonetic vowel "O" and "E". VojnosanitPregl 2015; 72(Pt 2):123-31.

15. Ismail $\mathrm{YH}$, George WA. The consistency of the swallowing technique in determining occlusal vertical relation in edentulous patients. J Prosthet Dent 1968; 19(Pt 3):230-6.

16. Shanahan TEJ. Physiologic vertical dimension and centric relation. J Prosthet Dent1956; 6:741-7.

17. Hernández R.Métodos para el análisis cuantitativo del electromiograma.Rev Cubana Invest Bio- med 1996; 15(Pt 2). [Citado 26 Julio 2019] http:// scielo.sld.cu/scielo.php?script=sci_arttextEpi$d=$ S0864-03001996000200007Elng=es.

18. Garnick J, Ramfjord SP. Rest position: an electromyographic and clinical investigation 1962; 12(Pt 5):895-911

19. Rugh JD, Drago CJ. Vertical dimension: a study of clinical rest position and jaw muscle activity.J ProsthetDent1981;45(Pt 6):670-5.

20. Michelotti A, Farella M, Vollaro S, Martina R. Mandibular rest position and electrical activity of the masticatory muscles. J ProsthetDent 1997;78(Pt $1): 48-53$.

21. Manns A, Miralles R, Guerrero F. The changes in electrical activity of thepostural muscles of the mandible upon varying the vertical dimension.J Prosthet Dent 1981;45(Pt 4):438-45.

22. Manns A, Valdivieso C, Rojas V,Valdés C,Ramírez V. Comparison of clinical and electromyographic rest vertical dimensions in dolichofacial and brachyfacial young adults: A cross-sectional study. J Prosthet Dent 2018; 120(Pt 4):513-9.

23. Fuentes R, Navarro P, Curiqueo A, Ottone NE. Determination of mandibular border and functional movement protocols using an electromagnetic articulograph (EMA). Int J ClinExp Med 2015; 8(Pt 11):19905-16.

24. Fuentes R, Saravia D, Arias A, Lezcano MF, Dias $F$. An innovative method to analyse the range of border mandibular movements using 3D electromagnetic articulography (AG501) and MATLAB. Biomed Research 2017; 28(Pt 9): 4239-47.

25. Kuruvilla M, Murdoch B, Goozèe J. Electromagnetic articulography assessment of articulatory function in adults with dysarthria following traumatic brain injury. BrainInj 2007; 21(Pt 6):601-13.

\section{CORRESPONDENCIA:}

Prof. Dr. Ramón Fuentes Fernández, Centro de Investigación en Ciencias Odontológicas (CICO), Facultad de Odontología, Universidad de La Frontera. Av. Francisco Salazar 01145, Temuco, Chile. Código Postal: 4811230. E-mail: ramon.fuentes@ufrontera.cl - Teléfono: 005692596902. 